\title{
An essay on the nominal vs. real definitions of aging
}

\author{
Aleksei G. Golubev
}

Received: 8 April 2021/Accepted: 31 May 2021/Published online: 6 June 2021

(C) The Author(s), under exclusive licence to Springer Nature B.V. 2021

\begin{abstract}
In the current literature, the definitions of aging range from relying on certain sets of distinctive features at the molecular, organismal, populational and/or even evolutional levels/scales to declaring it a treatable disease and, moreover, to treating aging as a mental construct rather than a natural phenomenon. One reason of such a mess may be that it is common in the natural sciences to disregard philosophy of science where several categories of definitions are recognized, among which the nominal are less, and the so-called real ones are more appropriate in scientific contexts. E.g., water is, by its nominal definition, a liquid having certain observable features and, by its real definition, a specific combination (or a product of interaction) of hydrogen and oxygen atoms. Noteworthy, the real definition is senseless for people ignorant of atoms. Likewise, the nominal definition of aging as a set of observable features should be supplemented, if not replaced, with its real definition. The latter is suggested here to imply that aging is the product of chemical interactions between the rapidly turningover free metabolites and the slowly turning-over metabolites incorporated in macromolecules involved in metabolic control. The phenomenon defined in this way emerged concomitantly with metabolic pathways
\end{abstract}

A. G. Golubev ( $\bowtie)$

Department of Carcinogenesis and Oncogerontology,

N.N. Petrov National Medical Research Center of

Oncology, Saint Petersburg, Russia

e-mail:1xglbv@rambler.ru controlled by enzymes coded for by informationstoring macromolecules and is inevitable wherever such conditions coincide. Aging research, thus, is concerned with the elucidation of the pathways and mechanisms that link aging defined as above to its hallmarks and manifestations, including those comprised by its nominal definitions. Esoteric as it may seem, defining aging is important for deciding whether aging is what should be declared as the target of interventions aimed at increasing human life and health spans.

Keywords Aging - Metabolism · Evolution · Philosophy of science

Philosophy of science is as useful to scientists as ornithology is to birds

Richard Feynman

A knowledge of the historic and philosophical background gives that kind of independence from prejudices of his generation from which most scientists are suffering. This independence created by philosophical insight is-in my opinion-the mark of distinction between a mere artisan or specialist and a real seeker after truth. Albert Einstein as cited by Laplane et al. (2019). 


\section{Introduction}

Several recent publications, including the one entitled "What if there is no such thing as aging?" (Cohen et al. 2020a), have brought out an astonishing trend: the mere existence of biological aging is being questioned. The title reiterates the famous proposition "There is no such thing as aging and cancer is not related to it" (Peto et al. 1985), and the paper suggests that the same is relevant to the other age-associated conditions: "...we are not studying a single biological phenomenon, but an assortment of loosely related processes that we find convenient to lump together"; ultimately, “. ... the concept of aging does not reflect any underlying biological reality" (Cohen et al. 2020a).

Being intentionally provocative, the paper (Cohen et al. 2020a) does however reflect the trend, which is developing, paradoxically, in parallel with the increasing prevalence of aged people in the world and with the recently emerged discipline called "geroscience" (Kennedy et al. 2014; Kaeberlein 2017; Blagosklonny 2021). According to the geroscience agenda, the best way to combat the most prevalent age-associated conditions, such as atherosclerosis, cancer, type II diabetes and neurodegeneration, is to target their common risk factor rather than each of the conditions specifically. The common risk factor is aging.

Apart of that the very practice of piling up of newly invented scientific disciplines and respective terms is questionable (Hayflick 2021), a problem with the geroscience agenda relates to the feasibility of evaluating the benefits of its implementation. The benefits of targeting of a disease may be evaluated, based on its commonly accepted definition (diagnostic criteria), as a decrease in the incidence of cases recognized according to this definition. Can we decrease the incidence of aging otherwise than by making people dead before they get old?

Any answer to this question depends on the criteria used to distinguish (i) aging from all the rest that may occur to living bodies and (ii) living bodies from all other kinds of bodies, in other words, on the definition of (biological) aging. The lack of consensus on the definition of aging is long recognized (Hayflick 2007) and has recently been highlighted in the paper (Cohen et al. 2020b) presenting the results of asking several basic questions about aging to recognized authorities in aging research. Answers to each of the questions differed up to antipodal extremes. One of the reasons of this discrepancy is suggested to stem from the different scientific backgrounds of the respondents and thus from the associated differences between the bases of their ideas about aging. The backgrounds look so diverse that a common objective basis for such ideas is unfeasible; therefore, "...aging is not a unitary phenomenon" (Cohen et al. 2020a).

Anyway, such ideas usually include certain definitions of aging.

A definition of aging may indeed be such that it will make its definiendum nonexistent. This is true, e.g., for aging defined as an evolved program of the gradual deterioration of living bodies for finally making them dead at a certain age. Although mechanisms that can terminate the life of living bodies of a certain kind under certain circumstances, including achieving a certain age, are plausible, and such cases have been reported, e.g. (Diamond 1982), there is no biological benefit to be supported by natural selection in a gradual deterioration starting long before the emergence of such circumstances, and there are other reasons to believe that aging is not programmed (Kowald and Kirkwood 2016).

Formulating any definition is a theoretical matter, so as understanding the meaning of the term "definition", which too should be ascertained (e.g., differentiated from explanation), to start with. This extends the discussion to the issues, such as relationships between definitions, explanations, concepts, meanings, models, and theories, usually delegated for consideration to philosophers. In particular, the arguments against the reality of aging (Cohen et al. 2020a) are largely based on linguistic and epistemological considerations, which look strikingly reminiscent of the linguistic relativity hypothesis (see e.g. (Casasanto 2016)) and the conceptual metaphor theory (see (Kövecses 2020)).

Several kinds of definitions are distinguished in philosophy of science (Gupta 2019). The associated discussions are too verbose, as it is common for philosophy, to be dwelt upon in a paper intended for a biological audience. An interesting question nevertheless is which of the kinds is the most appropriate for a useful definition of (biological) aging? The next question is then "useful for what?" For example, "a desperate need for a biomedically useful definition of aging" has been proclaimed (de Grey 2013). Does this mean that a definition of aging appropriate for 
biogerontology is not necessarily useful in biomedicine, or a definition suitable in gerontology is useless in geroscience?

The applicability of different kinds of definitions to aging may be explored based on the recent paper entitled "Defining aging" (Lemoine 2020), which may serve as a neat canvas for discussing the issue of how aging may be defined. The very emergence of this paper is a token that this issue has come of age. Another token is the explicit refusal to define aging while discussing it, as e.g. in (Rando and Wyss-Coray 2021). Instead, the authors focus on the manifestations of aging. The manifestations of what?

\section{Nominal vs. real definitions}

Based on several scores of definitions found in the most highly cited (that is the most representative and influential) papers on aging, the following features commonly used to define aging have been distinguished by Lemoine (2020): (1) structural damage, (2) functional decline, (3) depletion $<$ of a reserve required to compensate for the decline $>$, (4) typical phenotypic changes or their cause, and (5) increasing probability of death. Noteworthy, "these characteristics are not really five definitions of aging, but rather five defining features of aging" (Lemoine 2020). This remark attributes the above inventory to the kind of definitions that in philosophy of science is known as "nominal" and is opposed to "real" (Gupta 2019). The opposition may be illustrated with the substance named water in English.

By its nominal definition, water is a colorless and odorless liquid having defined specific gravity, viscosity etc. at a certain temperature and certain melting and boiling temperatures at a defined pressure. Other characteristics, such as absorption spectrum and refraction index may be added for the sake of reliability.

By its real definition, water is a compound comprised of two hydrogen atoms and one oxygen atom connected in a certain order.

The real definition may need additional explanations, especially for those who are not familiar with the concepts of atom and chemical bond. However, the real definition can convey the invariant essence of its definiendum, whereas the nominal definition only tells about the distinctive features of its definiendum, which may be accessible to a specific observer under specific conditions. Noteworthy, the observable features, at least some of them, may be inferred from the real definition by appropriate computations based on certain general physical principles and constants.

Another example is COVID-19. The observable features of this disease are so variable that the nominal definition is hardly possible. According to the real definition, COVID-19 is the symptomatic infection caused by SARS-CoV2 coronavirus (the product of interaction between the virus and human body, in terms of the definition by genesis-see below). However, this real definition is senseless for those who are ignorant of viruses.

The nominal definition of water is almost irrelevant to water in living cells, whereas the real one is highly relevant. Generally speaking, the real definitions are more appropriate for science, whereas the nominal ones, for household. Can there be a real definition of aging?

Another concern with the five features to be included in a nominal definition of aging (there may appear to be more features or some may prove to be superfluous) is that the first four items are related to individual bodies, whereas the last one, to the populations or cohorts of such bodies. Interestingly, some authors, e.g. (Faragher 2015), associate aging with a particular, i.e. exponential, mode of increase in mortality rate.

Still another concern is that all the items are as well applicable to diseases, especially endogenous chronic conditions. The issue of whether it is reasonable or useful to equate aging to disease is hotly debated (Bulterijs et al. 2015; Chmielewski and Strzelec 2020; Faragher 2015; Franceschi et al. 2018; Golubev 2018a; Zhavoronkov and Bhullar 2015; Rattan 2016; Hayflick 2021). Remarkably, the proponents of equating introduce one more, and non-scientific, dimension into the discourse: equating is justified by that without it there will be no incentives for investments into the projects of using drugs for treating aging as a disease under FDA approval (Kennedy and Pennypacker 2014). Defining aging as the sum of all age-associated diseases (Blagosklonny 2021) may probably be useful in the practice of lobbying such projects.

Several other concerns have been articulated by Lemoine (2020) to justify the proposition that "the most promising approach is to derive the definition $<$ of aging $>$ from a physiologically updated 
version of the theory of the evolution of aging". It has been suggested earlier by Martin (1996) that "Aging can be defined as a set of phenotypes that have escaped the force of natural selection". It is now generally accepted by evolutionary biologists that the chances to escape the force of natural selection increase with increasing the age after which a phenotype developes. This is because the force decreases as far as fewer organism, even if they were not aging or senescing, would be allowed to survive to later ages by the extrinsic causes of death (Hamilton 1966; Flatt 2012).

An important caveat concerning the above Lemoin's proposition is: "However, it is strange that the evolutionary theory of aging currently makes no reference to relevant findings of molecular biology, which, in turn, largely ignores evolutionary biology"; "...therefore, the most promising approach is to derive the definition from a physiologically updated version of the theory of the evolution of aging" (Lemoine 2020). Similar caveat has been articulated in (Golubev 2009). In the present essay, this approach to aging will be supported in its principle and modified in its embodiment.

The evolutionary aspect of aging brings the present discussion to a variant of the real definition known as definition by genesis. This attitude to definitions is most familiar to those who teach mathematics (Pimm 2014). For example, the right circular cylinder may be defined as a solid figure generated by rotating a rectangle round one of its sides rather than a combination of different elements including the base, the top, and the side surface.

This example may seem too abstract to be of any value for defining such a substantial phenomenon as aging; however, the above real definition of water may be construed as a definition by genesis (water is what is generated by heating two molar parts of hydrogen and one molar part of oxygen), and anyway the illustration may be of service as a sort of wireframe to fill it with substantive theorizing.

\section{Physics as a paragon}

Paragons of theorizing are usually searched for in physics. While being aware of all doubts and subtleties concerning the relationships between physics, chemistry and biology—see e.g. (Dagher and Erduran 2014)—let us still consider a basic physical notion, such as "work", as a paragon. Notably, the meaning of this physical term is not the same as that of its parent colloquial word (see, e.g., the Merriam-Webster Dictionary), from which the term was derived as late as in 1826 by Coriolis based on his experience in the early steam engines. (By corollary, the meanings of the biological term aging and the colloquial word aging need not be quite congruent.) In the physical discipline mechanics, the meaning of the term work may be expressed with a definition like the one found in Encyclopedia Britannica: "...the work $W$ is equal to the force $f$ times the distance $d$, or $W=f d$. If the force is being exerted at an angle $\theta$ to the displacement, the work done is $W=f d \cos \theta$ ". Thus, in mechanics, work is the product of force, distance, and the angle between the directions of the force exerted and the distance passed. The angle defines how the system $\{f, d\}$ is organized. When there is force and no displacement caused by it, then there is no work. When there is displacement and no force applied to cause it (e.g. displacement by inertia), then there is again no work.

In spite of doubts whether there is such thing as aging, it is more common to believe that biological aging is quite pervasive, so as mechanical work is. Let us try to treat the biological term aging similarly to the physical term work. It should be mentioned parenthetically that the meaning of the term aging in biology is not the same as in physics, e.g. (Dyre 2018), as well as in the common sense.

It has been suggested earlier (Golubev 1996, 2009; Golubev et al. 2017a, b) that the driving force of aging is, in other words aging is generated by, the chemical reactivity of the rapidly turning-over small metabolites applied to the slowly turning-over macromolecules implicated in metabolic control. Based on this proposal and by analogy with the above physical definition of work, aging may be defined as the product of the chemical interactions between the free metabolites and the metabolites incorporated in macromolecules.

This definition is not self-evident at all and implies some prior knowledge not only about the organization of living bodies but also about the chemical properties of its constituents, the properties being usually ignored by evolutionists, zoologist, geneticists, psychologists, demographers and other participants of discussions of the essence of aging. This is not to mention that laypeople are usually familiar with the accumulation 
of damage, decline in performance, depletion of reserves etc. much better than with organic chemistry. Thus, explanations for this definition are needed. However, the need for explanations of definitions is so pervasive that relationships between definitions and explanations is the matter of another lively, and everlasting, debate in philosophy of science, e.g. (Lewis 1970). The above definition of the mechanical work was too derived based on some prior knowledge of the properties of steam and the design of steam engines, which was far from being commonly known at the time of their origin from the precursory devices.

\section{The evolutionary justification for introducing chemistry into the real definition of aging}

Upon the origin of life from the precursory physicochemical world, the mess of spontaneous chemical reactions between small molecules, such as aldehydes, cyanides, thiols etc., was gradually transformed into ordered metabolic pathways or networks arranged by the catalytic activities of macromolecules ranging from ribozymes to protein enzymes (Muchowska et al. 2019; Ralser 2018; Tran et al. 2020; Wołos et al. 2020), which made use of those of the chemical potencies of small molecules that were beneficial for the survival of the primordial living entities. However, all other potencies did not just disappear. Although the space of chemical possibilities within proto-cells was becoming increasingly emptied outside of fluxes channeled by enzymes, the non-enzymatic interactions determined by the purely chemical properties rather than by the biological suitability of the interacting molecules persisted there thus constituting a sort of chemical heritage, which could yield useless and even noxious products. Interestingly, most of such non-enzymatic processes involve chemical reactions honored with proper names, such as Haber-Weiss reaction, Michael addition, Pictet-Spengler reaction, Schiff base formation, Amadori rearrangement, Mannich reaction, etc. (Golubev 1996; Golubev et al. 2017a, b), just because they are typical and realizable under even mild conditions when the respective reactant molecules collide.

When the molecules of a highly reactive spontaneously formed metabolic byproduct, such as methylglyoxal, collide with, say, amino group moieties of other molecules, the resulting stable covalent adducts will be kept at a stationary level by turnover if the amino groups are comprised by small rapidly exchangeable molecules; however, if turnover is incompatible with the function performed by macromolecules, such as those (i.e. DNA) that store the information about the structure of the catalysts (i.e. proteins) involved in organizing the metabolic fluxes, then the results of such collisions will accumulate and progressively compromise the functions of the proteins that are long-lived and/or are produced according to instructions derived from the damaged DNA.

Such gradual deterioration is inevitable in systems that exist due to energy and other resources provided by the rapid turnover of sufficiently reactive small molecules (metabolism), which is controlled by slowly turning-over macromolecules whose proper structure in the course of their turnover is reproduced over and over again due to the macromolecules that store the reference information about this structure (heredity) and therefore are exempted from turnover, which is incompatible with their information-storing function. Aging has emerged concomitantly with the emergence of such intermolecular relationships, i.e. it is intimately associated with the origin of life. This means that "The moulding of senescence by natural selection" (Hamilton 1966) may relate only to the contribution of the natural selection to shaping the patterns of senescence but not to the emergence or origination of this independent factor of the evolution of life (Golubev 2009). That is what justifies the above contention that "the evolutionary theory of aging currently makes no reference to relevant findings of molecular biology".

The turnover of the information-storing macromolecules is possible, however, at the level of populations of living entities due to their self-reproduction, which compensates for their disintegration (death). This makes it possible to keep the level of damage of information-storing macromolecules at a tolerable stationary level in a population. However, this does not preclude damage accumulation in the slowly renewable components of the individual bodies that comprise such a population. Aging will be apparent in such bodies if the half lifetimes of some of macromolecules comprised by them are comparable with the half lifetime of the bodies, which is defined by the rate of their turnover.

Thus, the chemical reactivity of metabolites applied in living systems to their components whose functions 
are incompatible with rapid turnover is sufficient to compromise progressively the viability of such systems, that is to generate their aging, and nothing else is indispensable for the genesis of aging. There may be, however, additional nonobligatory factors, which could emerge later, such as telomere attrition in eukaryotic cells or memory cells buildup in the immune system of mammals. Such newly emerged factors could be spared by natural selection just because they did not add significantly to the mortality patterns resulting from the preexisting aging. Moreover, telomere attrition may occur due to telomeric DNA damage by free radical species generated as byproducts of metabolism even in non-replicating cells whether there is no DNA replication problem at work. Even the complete elimination of the DNA end replication problem will not stop aging. Indeed, aging is experienced by organisms, such as nematodes and rotifers, whose soma comprises only post-mitotic cells where DNA is exempt from replication.

Aging can be stopped only by the complete elimination of either metabolism or informationstoring macromolecules, that is, by the elimination of that what makes life alive. Upon the lack of any of the two sides of aging defined as the product of chemical interactions between metabolites and macromolecules, there is no aging. Inanimate entities, such as automobiles or glasses do deteriorate as time passes, but they do not age because there is no metabolism in them. Living entities without unrenewable components do not age, no matter how active metabolic processes are there. For example, hydras are able to avoid aging (Schenkelaars et al. 2018) because all cell populations in hydras are renewable by cell turnover. By contrast, any of the constituent individual cells in the body of a hydra does age to the extent allowed by the time provided by cell lifespan, which is limited by cell turnover rate. A population of organisms that do age, because they contain unrenewable components, does not age, because its constituent individuals are renewable by their turnover.

Both metabolism and nonrenewable components are present in bacteria. Individual bacterial cells do age (Moger-Reischer and Lennon 2019; Yang et al. 2019), although not all of the alleged nine hallmarks or seven pillars of aging (López-Otín et al. 2013; Kennedy et al. 2014) are unquestionably relevant to bacteria.

\section{The real definition of aging by genesis vs. manifestations of aging used in its nominal definitions}

The assortment of the primary producers of the endogenous damage to macromolecules is commonly believed to comprise primarily the reactive oxygen and nitrogen species (Harman 1992; Droge 2003; Barja 2014; McCann et al. 2005), and the reactive carbonyl-containing molecules, including reducing sugars, such as glucose (Lyons and Jenkins 1997; Semchyshyn 2014). This assortment has recently been enriched with the reactive carbamyls (Gorisse et al. 2016) and thioethers (Simic et al. 2015) and may be updated further (Golubev 2019b). Upon comparing a metabolic map with a comprehensive course of organic chemistry, a lot of chemically caused leaks and short circuits may be pinpointed within and between the pathways controlled by enzymes. Most of these leaks and short circuits may be attributed to much fewer typical chemical interactions between the reactive moieties of metabolites. However, the combinatorial diversity of such typical interactions is immense (Lerma-Ortiz et al. 2016) thus generating a sort of pressure at work within cells.

The position advocated here implies that this pressure is generated by aging consistent with its real definition. All other definitions are nominal and relate only to the particular manifestations of aging defined in the real way. This statement is illustrated in Fig. 1.

Because aging works in organized systems, its results are shaped by their organization, so as steam pressure may drive rotation, elevation, expansion, rocking or whatever else, depending on the design of a steam engine and its particular details. An obvious difference between an engine and a living cell, in this regard, is that, in the former, a cause will produce its effect for sure, whereas in the latter, most cause-effect relationships are probabilistic. In a cell population, not all cells but only a certain proportion of them change their fates in response to a challenge. This is not because different cells feature different fixed sensitivities to the challenge, but because the inherent stochasticity of intracellular processes implicated in cell responses to a defined challenge will make any outcome probabilistic (Golubev 2012). In particular, intracellular molecular processes are organized so that aging as the product of chemical interactions between metabolites and macromolecules increases the 


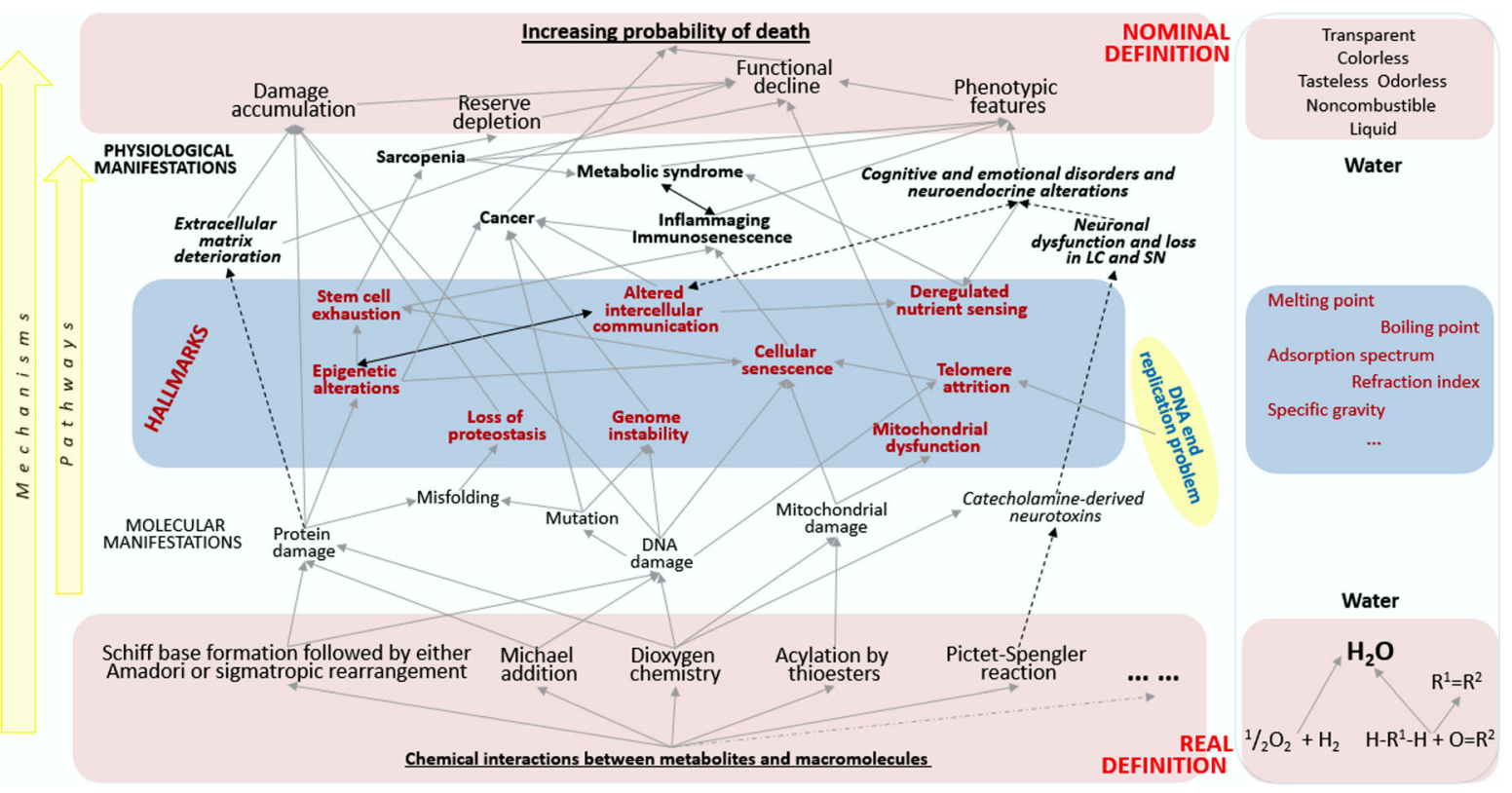

Fig. 1 Relationships between the real and nominal definitions and the hallmarks of aging. The nominal definition (the upper shaded panel) includes all items listed by Lemoine (2020). The hallmarks (the middle panel) are as defined by Lopez-Otin et al. (2013). The real definition (the lower panel) is as outlined here in the main text. Arrows show some of the most obvious gross cause-effect relationships, which may include intermediate steps and be influenced by independent factors. An important independent factor is the DNA-end replication problem, which is unrelated to the basic real definition of aging. Some of the

probability of cell transition to the senescent state, as it occurs, e.g. as a result of methylglyoxal interactions with DNA (Larsen et al. 2012). The central nervous system is organized so that some neurons use catecholamines as neurotransmitters. The high chemical reactivity and the resulting cytotoxicity of catecholamines (Goldstein et al. 2014), which is realized, in particular, via the Pictet-Spengler chemistry (Golubev 1996; Golubev et al. 2017a; Peana et al. 2019) is relevant exclusively to the catecholaminergic neurons, which are concentrated in specific brain compartments. Therefore, the age-associated increase in the probability of neuron death is the highest in such compartments, e.g. the Locus coeruleus and Substantia nigra, which age in a peculiar way associated with neuromelanin accumulation (Hassani et al. 2020; Krashia et al. 2019; Wang et al. 2019). The involvement of projections of these neurons in the regulation of specific cortical functions shapes the cognitive and emotional manifestation of aging (Jacobs et al. 2019; relationships may be reciprocal, as illustrated with the thicker double-arrowhead lines. Some may involve factors operating at higher levels to produce effects at lower levels. Dashed lines show the relationships that are relevant to the whole-body level. LC and SN stand for Substantia nigra and Locus coeruleus, which are the brain structures that are directly affected by the high chemical reactivity of dopamine and norepinephrine, respectively. The consequences of this reactivity may extend as far as to epigenetic alterations in remote organs

Weinshenker 2018). The involvement of catecholamines in the central regulation of endocrine functions shapes the neuroendocrine manifestations of aging (Dilman et al. 1986), including increases in the production of several hormones, such as gonatodropins, in mammals but not in living things organized otherwise.

This attitude to aging may seem a sort of brutal reductionism. There is no reductionism in it actually, because the phenotype of aging is not claimed here to be reducible to chemistry or deducible from only chemistry and nothing else. The chemical effects look as they do in living bodies only because the bodies are organized as they are. Their organization is such that systems referred to different levels of organization may interact to produce the whole-body responses to the primary local age-associated changes. Such responses include compensation for the primary ageassociated deficits and mitigating the effects of the external factors that can accelerate or aggravate aging. 
Obviously, when something at a lower level is affected by a response of something else at a higher level to what the former something does, then the result of the interaction between the two levels cannot be derived exclusively from what is known about the lower level. Both levels and everything in between must be taken into account. Responses of higher-level cellular systems to transient stresses acting at a lower molecular level may enhance the abilities of living bodies to control the ever-present inner chemical forces that are responsible for the primary driving force of aging. Such responses are likely to contribute to the phenomenon known as hormesis (Rattan 2012). In particular, the effects named mitohormesis and glycohormesis, which are mediated by transient increases in ROS and methylglyoxal, respectively, have been described, and some signaling pathways involved in these effects have been determined (Kold-Christensen and Johannsen 2020). At the whole-body level, such systems may comprise feedback loops spanning through different levels of organization, including the higher integrative functions performed by the neuroendocrine system. For example, aging in mammals is associated with increases in the actual production of gonadotropins upon the background of the aging associated decrease in the maximal secretory capacities of pituitary gonadotrophic cells. This increase in gonadotropin secretion, which occurs in response to the primary decrease in the steroidogenic capacity of sex glands, may be additionally exaggerated by the reduced function of the central neuroendocrine mechanisms that mediate the negative feedback between the activities of gonadotrophic and steroidogenic cells (Dilman et al. 1986). Such multidirectional yet coordinated aging-associated physiological changes may look as a programmed process. However, such quasi-program is not the projection of the developmental program into the adult state, as it has been suggested (Blagosklonny 2006), but rather is an emergent phenomenon.

Delineating the pathways by which aging defined by its genesis acts in specific components of living bodies to generate its emergent manifestations at the higher levels of organization, that is deciphering the mechanisms of aging, is what aging research is primarily about. These manifestations may be contributed to by other factors, such as telomere attrition due to the DNA end replication problem. However, this problem itself could not emerge without prior emergence of metabolic processes and macromolecules that control them and thus it cannot exist separately from aging defined as it is done here, whereas aging can be without telomere attrition, e.g., in bacteria (Moger-Reischer and Lennon 2019; Yang et al. 2019) or in transgenic mice expressing telomerase in all of their cells (Tomás-Loba et al. 2008).

The same relates to the features of aging rated in (Kennedy et al. 2014; López-Otín et al. 2013) as its pillars or hallmarks.

Among these hallmarks, the "loss of proteostasis" includes protein misfolding and aggregation, in particular amyloid formation, which may be interpreted as resulting from the chemical capability of amino acid residues for other chemical interactions besides those involved in proper folding. Moreover, the chemical modifications of amino acid residues by metabolic intermediates and byproducts may accelerate misfolding in the proteins that otherwise are quite stable (Taghavi et al. 2016).

The aging-associated "epigenetic alterations" may be either primary, i.e. resulting from the direct chemical modification of chromatin, or reactive, i.e. associated with the changes in gene activity that occur in response to the changes in body functions that are caused by the primary chemical damage to its structures, the changes in body functions being brought about via pathways defined by body design, and the changes in gene activity being produced by the enzymatic methylation of DNA, which is included in body design.

The causes of the aging-associated "mitochondrial dysfunction" are contributed to by the chemical properties of oxygen, including its preference for accepting electrons one by one rather than in pairs. Electrons transport via the respiratory chain is coupled to protons pumping out of mitochondria, which makes their inner milieu alkaline and thus favorable for the spontaneous acylation of amino groups by thioesters (Hong et al. 2016).

The "loss of genome integrity" may be, to a significant extent, attributed to the chemical modifications of nucleic bases, which may be transformed to mutations by DNA replication machinery. In this, as well as in a number of other respects, including the DNA end replication problem, the replicative machinery may be blamed to be a contributor to the imperfectness of living bodies, which is declared to constitute the ultimate cause of aging (Gladyshev 
2013). Upon expanding this outlook onto metabolites and proteins, they too may be judged as imperfect, as far as the former can make damage and the latter can be damaged. Indeed, a perfect metabolite must be such that it can do nothing without enzymes, and a perfect macromolecule must be immune to any chemical attacks by reactive metabolites. In this sense, most metabolites and macromolecules are terrifically imperfect. However, the imperfectness of a molecule is traceable to the excess of its chemical potentialities over those that are necessary and sufficient for its biological functions, and, since the potentialities are the same no matter where, to blame imperfectness for the adverse phenomenon of aging is the same as to claim that nothing and nobody is perfect in the real world, which is certainly the ultimate truth but hardly a revelation.

Importantly, putting imperfectness at the base of aging does not allow distinguishing the biological aging from the time-dependent deterioration of inanimate bodies, such as all sorts of machines. A perfect machine must not deteriorate. However, each kind of machines exhibits an increase in failure rate upon increasing operation time; moreover, such increases are often similar to exponential. Putting entropy increase at the base of aging (Calloway 1966; Samaras 1974; Hayflick 2007) is also applicable equally well to both animate and inanimate entities, whereas a meaningful definition of the biological aging must differentiate it from the deterioration of the second ones. This requirement is met by the definition suggested herein, which is based on metabolism and heredity, the two features that are essential for life and differentiate it from the nonliving world.

To understand why the particular form of deterioration, which is specific to living bodies in that it is attributed to the chemical interactions between metabolites and macromolecules, manifests itself as it does in particular organisms is the same as to understand their normal organization and functions, including the chemical properties of the material involved in these functions. The scientific quest for control over aging contributed tremendously to understanding the design of living bodies, so as the alchemic quest for the Philosophers' Stone contributed to the origin of science. The latter consideration provides grounds for treating the geroscience agenda as excusable and useful in spite of that it renders the agenda unfeasible as well.

\section{What the allegedly anti-aging interventions can really do if there is such thing as aging consistent with the real definition thereof?}

The assumption that the primary driving force of aging is constituted by spontaneous chemical interactions directly suggests that aging will be accelerated by promoting such interactions and decelerated by inhibiting them. Indeed, the importance of oxygenderived free radicals for aging was recognized, in the form of the free-radical theory of aging (Harman 1992), based on the observation that aging is accelerated by ionizing radiation, which generates hydroxyl radicals from water. The importance of the nonenzymatic modification of proteins and DNA by glucose for aging is supported by the observations that aging is accelerated in rodents fed with diet where glucose is substituted for with galactose, which is a more potent glycation agent (Azman and Zakaria 2019). In such cases, the acceleration of aging may be attenuated by antioxidants or antiglycation agents, respectively. As to a non-accelerated normal aging, numerous trials of antioxidants as anti-aging agents have been carried out to meet some success in some specific cases only. The current consensus is that none of the interventions believed to reduce reactive oxygen species (ROS) production, enhance their scavenging, block their interactions with macromolecules, or repair the resulting damage have proved to produce robust anti-aging effects (Sadowska-Bartosz and Bartosz 2014). The same is true for the attempts to combat aging with glycation inhibitors or deglycation enhancing agents, which however do ameliorate some specific manifestation of aging (Sadowska-Bartosz and Bartosz 2016).

There may be several explanations for these failures. One is that ROS species perform specific biological functions, and damage produced by them is a byproduct of performing these functions (Sies and Jones 2020), which may be compromised by interventions aimed at minimizing ROS levels and/or effects. Indeed, enzymes specialized in superoxide generation are known. The effects of the respective genes are both beneficial, as far as they are mediated by superoxide, and detrimental, as far as hydroxyl radicals are generated from superoxide via HaberWeiss reaction. This provides reasons to regard the effects of these genes as antagonistically pleiotropic and thus points out another aspect of the relevance of 
the definition of aging suggested herein to the evolutionary theories of aging (Golubev et al. 2017b; Golubev 2019b).

One more explanation may be that different reactive metabolites compete for common targets, e.g. sulfhydryl and primary amino groups are targeted by both ROS and reactive carbonyls. Therefore, inhibiting some specific chemical reactions will spare targets for some other reactions and thus will influence only the composition but not the amount of damage.

Still other explanation is based on that mitigating the effects of an endogenous damaging factor consumes resources, which are thus distracted from performing other vital functions, including mitigating the adverse effects of other endogenous factors. For example, methylglyoxal (MGO) detoxification is associated with reducing equivalents consumption.

It is also important that the biochemical machinery insides cells must be tuned in evolution to the unavoidable presence of damaging factors, such as MGO; therefore, their reduction enforced by an intervention from the outside may be associated with functional disarrangements. For example, among the protein targets of the chemical reactivity of MGO, the MGO-modified protein KEAP features a decreased ability to deactivate the transcription factor NRF-2, which enhances the expression of the enzymatic protective means against oxidants and reactive carbonyl species (Bollong et al. 2018). Therefore, a decrease in MGO level below optimum is associated with a corresponding decrease in cell ability to counteract the endogenous chemical damage. Increased MGO production resulting from an experimental modification of threonine catabolism has been associated with enhanced autophagy and increased lifespan in several animal species (Ravichandran et al. 2018). Reduced brain MGO is associated with neurological impairments in mice (Wu et al. 2018).

Moreover, even if an intervention is found to increase the lifespan of the organisms under study, this does not necessarily mean a deceleration of aging, as it will be shown below. This brings the discussion to the level of populations or cohorts, which cannot be ignored because the nominal definitions of aging usually imply that aging increases the probability of death, which makes sense only in populations. Some authors, e.g. (Flatt 2012; Golubev 2009, 2019a, b), even associate aging with specific, such as exponential, trajectories of increases in chances of dying upon increasing time of living.

This issue will be clarified with a mental experiment. Consider a situation of the need to jump over two meters-wide water-filled trenches in order to save life from some crawling predators. The metabolites involved in producing energy for exercising this ability are capable of chemical interactions yielding noxious byproducts, such as methylglyoxal in glycolysis and/or ROS in oxidative phosphorylation. The damage caused by such byproducts to the nonrenewable structures of the living bodies that have to cross the trenches in order to survive accumulates and gradually decreases the maximal capacity (potential) for exercising the jumping ability. The realization of any remaining potential for crossing the trenches can never be complete because there are always competing problems to deal with. Those bodies that at older ages are able to realize more of the remainder of their potential for crossing the trenches may do it better than those that at younger ages are able to realize less of their relatively higher potential. At an age of about 25 time units, almost anybody is able to cross a trench without much effort for mobilizing the available potential. At about 60 , only those can cross the next trench who have been trained in mobilizing the remainder of their potential or supplied with a substance that enhances the ability to mobilize the potential on emergency. However, no training or drug can help to exceed the potential, which decreases with increasing age. At about 90, nobody's potential for crossing trenches is high enough. Only an external help (e.g. an ambulance brigade) can bring one over a trench and let survive until meeting the next obstacle. May we think that the regular use of ambulance, training or performance-enhancing drugs slows down aging? The potential to overcome obstacles encountered on the way to further life decreases anyway, as may be illustrated with the age-specific track-and-field world records decreases upon increasing age (Marck et al. 2017).

This mental experiment may be elaborated with the assumption, which is prompted by the Strehler-Mildvan theory of aging (Strehler and Mildvan 1960), that trenches differ in their widths, wider trenches being less prevalent. Let the number of trenches decrease exponentially with increasing their width. Most trenches are so narrow that they are passed unnoticed at most ages, although even at an age of about 25 or 
younger there is a small chance to meet an unsurpassably wide trench. If the capability for surpassing trenches decreases in a linear fashion with increasing age, then the incidence of failures in surpassing trenches will increase exponentially upon aging, consistent with the Gompertz-Makeham (GM) law of the dependency of mortality rate on age:

$\mu(t)=C+\mu_{0} \exp (\gamma t)$,

where: $t$ is age, $\mu$ is mortality rate, $C$ is the ageindependent rate of mortality caused, e.g., by falling meteorites, $\mu_{0}$ is the initial mortality rate, which may be interpreted as the reverse of the initial viability, which decreases because of aging, and $\gamma$ may be interpreted as the rate of aging.

Using a geroprotector will help to surpass any next trench and thus postpone the moment of meeting an unsurpassable obstacle. If this effect does not depend on age, there will be a parallel shift of a survival curve to later ages. In terms of the GM law (or model), this means a decrease in $\mu_{0}$ whereas $\gamma$ will remain the same. It is likely, however, that the effect will decrease with aging because less of the potential to be realized is left as age increases. This means that the shift of the survival curve to later ages will be smaller in older animals, and this will make the downward slope of the curve increasingly steeper.

Differentiating between changes in lifespan caused by an increase in viability or a decrease in the rate of its decline, i.e. in the rate of aging, is illustrated in Fig. 2 where survival curves are constructed according to the GM law.

The curves in Fig. 2 are constructed by picking the GM parameters from ranges typical for mice. The maximal modeled lifespan is the age after which the proportion of survivors in an experiment with a defined initial number of mice would make the number of still living mice below unity. If the initial number of mice were 20 , their maximal lifespans (more rigorously, their mathematical expectations) in such cohorts would be defined by the interceptions of the survival curves with the upper thin line parallel to the abscissa. Clearly, the maximal lifespan may be extended by increasing the initial number of mice from 20 to 50. However, at cohort sizes above ca. 100, this effect must be negligible. In Fig. 2, the maximal lifespans in such cohorts correspond to the age at which survival curves apparently merge with the abscissa. Thus, it may be seen that equivalent extensions of the maximal lifespan from ca. 1000 days to about 1250 may be achieved by the interventions started at the age of about 200-400 days (as it is usual in experiments with mice) that result in either a decrease in only $\mu_{0}$ or a decrease in only $\gamma$. The same is true for lifespan extension to about 1430 days. Noteworthy, the survival curves run higher when $\mu_{0}$ is increased. This suggests that the mean or median lifespans must be higher and the health spans must be longer in such cases. It may be also seen that at an age of, e.g., about 900 days, the same 7- to 8-fold increment in survival may be achieved by either reducing $\gamma$ by ca. $30 \%$ or reducing $\mu_{0}$ fivefold. To appreciate the magnitudes of these reductions, it is worthy to consider that, in mice, $\gamma$ and $\mu_{0}$ estimates usually vary within a threefold range and as much as 100-fold, respectively.

An important conclusion from Fig. 2 is that an increase in life and/or health span is not necessarily associated with a deceleration or delay of aging. To define whether an increase in the initial viability (and the associated increased viability throughout lifespan) or a deceleration of the age-associated decrease in viability, i.e. of aging, is responsible for an increase in lifespan, it is helpful to analyze the shapes of the experimental survival curves according to the model chosen for the dependency of mortality of survival on age.

Attitudes to the GM law currently range from treating it as merely a convenient statistical model or tool for presenting survival data, as it is epitomized in (Stroustrup 2018), in which case most or the above reasoning is just empty, to believing that the law has a sound natural basis, which may be obscured by the default assumption that the age-associated decrease in viability is linear throughout the entire lifespan, which is not generally true (Golubev 2009, 2019a, b). The former approach does not imply any natural restrictions for changes in survival patterns and thus for the quest for a longer life. The latter one is more restrictive in this regard. Reasons to believe that the latter is true, including the argument that the exponential increase in mortality is derived from chemical kinetics and thus is a sort of another chemical heritage of biology, are disclosed in a series of papers (Golubev 2009, 2019a, b; Golubev et al. 2018) where arguments both pro and contra are discussed in detail unfeasible in the present context. 


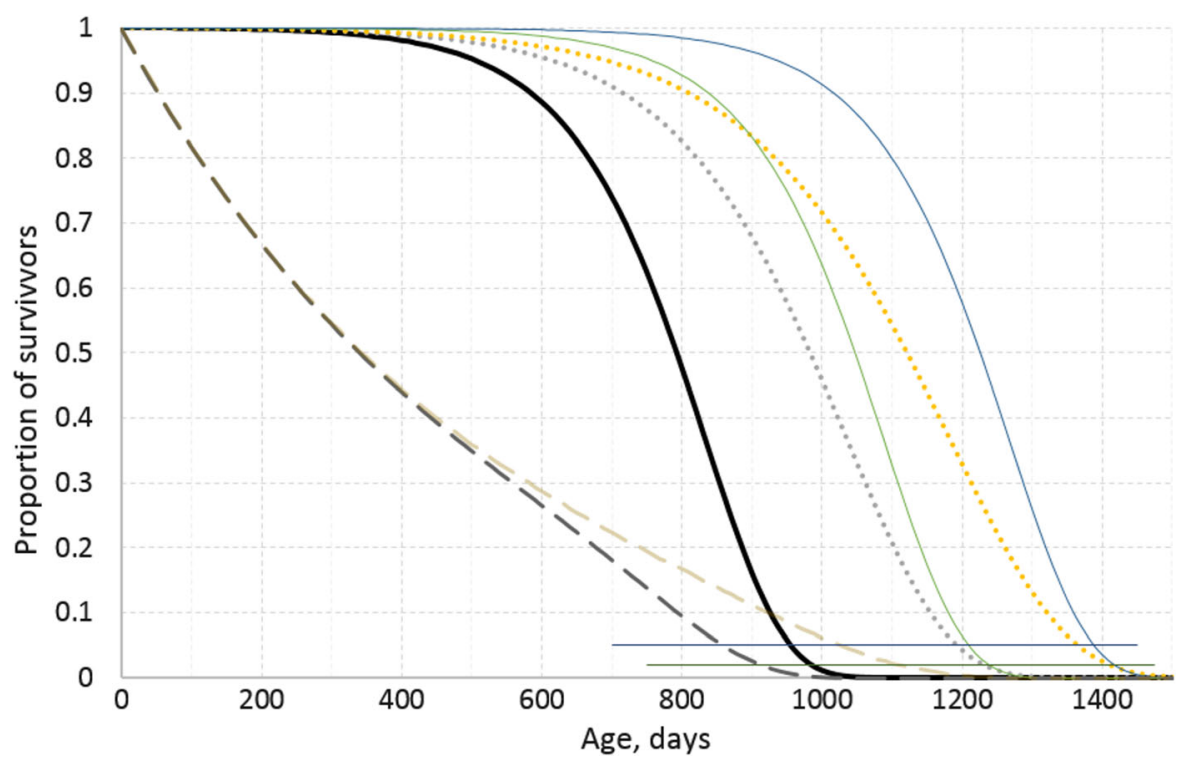

Fig. 2 Survival plots constructed according to the GompertzMakeham law. The values of its parameters are chosen from ranges typical for laboratory mice. The thick solid line is the reference plot $\left(\gamma=0.009\right.$ days $^{-1}, \mu_{0}=5 \times 10^{-6}$ days $^{-1}$, and $C=0$ days $^{-1}$ ). The dotted lines are constructed by decreasing $\gamma$ to 0.007 and 0.006 . The thin solid lines are constructed by reducing $\mu_{0}$ to $10^{-6}$ and $5 \times 10^{-7}$. The two dashed lines are constructed by adding $C=0.002$ days $^{-1}$ to the reference

To proceed with the imaginary situation considered here, it may be made closer to reality by assuming that obstacles to further life include not only trenches but also fences having different heights. Life may be interrupted by either a trench or a fence. The type of the obstacle that may turn out to be unsurpassable is unpredictable, but it is reasonable to assume that life interruption by a trench is less likely for those who trained their legs, and by a fence, for those who trained arms, there being a tradeoff between the two types of training. Can we think that training sessions or drugs that enhance the abilities to mobilize the potential for jumping or climbing slow down aging?

Why not, if physical training is known to reduce the risks of not only accidental stumbles and falls, the incidence of which increases upon aging, but also of cardiovascular, oncological, neurodegenerative and other age-associated conditions? It is not because only the realization of the potential to avoid or withstand such risks is enhanced. However, no realization can exceed what is supposed to be realized, which decreases with increasing age. This is also true for the geroprotector drugs claimed by some authors to conditions (the darker line) or conditions modified by decreasing $\gamma$ to 0.007 (the lighter line). The two thin lines parallel to the $\mathrm{X}$-axis denote survival rates equal to 0.05 or 0.02 . Such rates correspond to the ages at which only the last one of initially 20 or 50 survivors, respectively, remains alive. These ages correspond to the mathematical expectations of the maximal lifespans achievable in the respective cohorts

mimic the effects of physical activity (Muise et al. 2019; Hawley et al. 2021) and/or of dietary calorie restriction (Martel et al. 2021). Moreover, any such drug can target only a part of molecular interactions actuated by the two physiological intervention. Mimetics can only mimic, that is they cannot reproduce fully the mimicked interventions and thus cannot perform better than the latter can (Golubev 2018b, 2020; Rattan 2020).

The only intervention known to reliably slow down the age-associated decrease in the potential to withstand the causes of death and thus to make the downward bends of survival curves less steep (dotted lines in Fig. 2), rather than to produce parallel shifts of the curves towards later ages (thin lines), is calorie restriction. However, there is an important caveat regarding this intervention. Under normal (control) laboratory conditions, food is available ad libitum. Under natural conditions, the availability of food ad libitum, without physical efforts and associated energy expenditures for foraging, in not normal. Therefore, the standard laboratory conditions are abnormal in comparison with those that have 
"molded" a given species by natural selection and to which the functions of a body are tuned. Placing mice under control laboratory conditions may be thought of as a treatment that accelerates the age-associated increase in mortality rate. When, upon the comparison of two groups of animals, the one treated in a way known to accelerate mortality rate increase upon aging (e.g., the use of the free-radical inducer paraquate to treat fruit flies or of the glycation agent galactose to treat mice) is considered as control, and the other, which is not treated in this way, is considered as experimental, then the number of successful cases of experimental deceleration of aging will dramatically increase. When, upon the comparison of a mutant featuring an accelerated aging and the wild type, the wild type is considered as a success in repairing the mutation, then lo and behold: there is a success in the deceleration of aging. Calorie restriction may well be among such cases of the normalization of an increased aging rate rather than the deceleration of the normal aging rate.

Calorie restriction is a norm under natural conditions. However, such conditions are usually associated with a high age-independent mortality. This situation is shown in Fig. 2 with the lighter dashed line. For comparison, the same age-independent (extrinsic) mortality is added (the darker line) to the situation of ad libitum food availability. It may be seen that, under sufficiently high extrinsic mortality, food excess makes little harm in terms of survival.

The putative geroprotector drugs advertised by geroscientists increase lifespan in the animals placed under conditions that are conventionally regarded as control but, actually, are abnormal and associated with accelerated aging. The drugs used under such conditions may improve the ability of the animals to mobilize their potential for survival, but they do not slow down the accelerated age-associated decrease in the potential. This is evident from analyses of the respective published survival curves: only the preexponent term of the Gompertz-Makeham law ( $\mu_{0}$ in Eq. 1) is reduced by any of the putative geroprotectors (Golubev 2019a, b). Moreover, increases in viability, which are reflected in decreases in the pre-exponent term, are usually associated with increases in the term that reflects the rate of aging ( $\gamma$ in Eq. 1). This effect, which is known as the Strehler-Mildvan correlation or the compensation effect of mortality, may be partly attributed to the mathematical artifacts of survival curve fitting (Tarkhov et al. 2017); however, the components that are irreducible to such artifacts may be distinguished in the correlation (Golubev 2019a, b).

\section{Conclusion}

In the final account, the question "What if there is no such thing as aging?" is the same as "What if there are no such things as metabolism and heredity?" The answer is "there will be no such thing as living".

Aging will be wherever metabolic processes involving the free metabolites are controlled by the metabolites incorporated in the slowly turning-over macromolecules. That is why aging may be defined as the product of chemical interactions between metabolites and macromolecules. All other definitions of aging actually relate to its particular manifestations, which may be or may be not there. The cause-effect chains to each of the manifestations or features of aging from their common root, which is aging defined as above, may have different lengths and directions (Fig. 1) making the impression that the manifestations are related but loosely. "Clearly, phenotypes of aging arise as a result of underlying molecular processes, but unlike genetic diseases that are unequivocally due to genetic mutations, there is no obvious or comparable starting point" (Rando and Wyss-Coray 2021). A logical starting point need not be mapped to a particular structure. The starting point advocated in the present essay is the set of chemical phenomena, which may indeed seem not as obvious as a single mutation is; however, the starting point, or rather the starting ground, as defined here is relevant to all living organisms, including the bearers of the mutation that may modify the conditions that shape the pathways of the sprouting of the effects that emanate from this starting point and project them to the observable manifestations.

By defining what aging is, the present discussion also suggests what aging is not. Aging is not a nonentity. It is not a disease. It is not a trait selected in evolution for bringing living things to timely death. Moreover, aging is not what could emerge for whatever end at some point of evolution by natural selection. It has emerged concomitantly with the emergence of life and has been thereafter an indispensable condition for the biological evolution to cope with. 
Esoteric as it may seem, defining aging is important in utilitarian terms for deciding whether aging should be the declared as the main target of interventions intended to increase human life span and quality. The definition of aging put forward in the present essay suggests that living prolonged by attenuating of aging is equivalent to aging prolonged by attenuating of living. It is doubtful whether such an objective is worth pursuing.

Acknowledgements The author is grateful to Prof. V.N. Anisimov (National Medical Research Center of Oncology, Saint Petersburg, Russia) for encouragement, reviewing the manuscript, and valuable advice.

\section{Declarations}

Conflict of interest The author declares no conflicts of interests.

\section{References}

Azman KF, Zakaria R (2019) d-Galactose-induced accelerated aging model: an overview. Biogerontology 20:763-782. https://doi.org/10.1007/s10522-019-09837-y

Barja G (2014) The mitochondrial free radical theory of aging. Prog Mol Biol Transl Sci 127:1-27. https://doi.org/10. 1016/b978-0-12-394625-6.00001-5

Blagosklonny MV (2006) Aging and immortality: Quasi-programmed senescence and its pharmacologic inhibition. Cell Cycle 5:2087-2102. https://doi.org/10.4161/cc.5.18. 3288

Blagosklonny MV (2021) The goal of geroscience is life extension. Oncotarget 12:131-144. https://doi.org/10. 18632/oncotarget. 27882

Bollong MJ, Lee G, Coukos JS, Yun H, Zambaldo C, Chang JW, Chin EN, Ahmad I, Chatterjee AK, Lairson LL, Schultz PG, Moellering RE (2018) A metabolite-derived protein modification integrates glycolysis with KEAP1-NRF2 signalling. Nature 562:600-604. https://doi.org/10.1038/ s41586-018-0622-0

Bulterijs S, Hull RS, Björk VCE, Roy AG (2015) It is time to classify biological aging as a disease. Front Genet 6:205. https://doi.org/10.3389/fgene.2015.00205

Calloway NO (1966) The role of entropy in biologic senescence. J Am Geriatr Soc 14:342-349. https://doi.org/10.1111/j. 1532-5415.1966.tb05595.x

Casasanto D (2016) Linguistic relativity. Routledge handbook of semantics. Routledge, New York, pp 158-174

Chmielewski PP, Strzelec B (2020) If ageing is a disease, then life is also a disease. Anthropol Rev 83:307. https://doi.org/ 10.2478/anre-2020-0017

Cohen AA, Legault V, Fülöp T (2020a) What if there's no such thing as "aging"? Mech Ageing Dev 192:111344. https:// doi.org/10.1016/j.mad.2020.111344
Cohen AA, Kennedy BK, Anglas U, Bronikowski AM, Deelen J, Dufour F, Ferbeyre G, Ferrucci L, Franceschi C, Frasca D, Friguet B, Gaudreau P, Gladyshev VN, Gonos ES, Gorbunova V, Gut P, Ivanchenko M, Legault V, Lemaître J-F, Liontis T, Liu G-H, Liu M, Maier AB, Nóbrega OT, Olde Rikkert MGM, Pawelec G, Rheault S, Senior AM, Simm A, Soo S, Traa A, Ukraintseva S, Vanhaelen Q, Van Raamsdonk JM, Witkowski JM, Yashin AI, Ziman R, Fülöp T (2020b) Lack of consensus on an aging biology paradigm? A global survey reveals an agreement to disagree, and the need for an interdisciplinary framework. Mech Ageing Dev 191:111316. https://doi.org/10.1016/j. $\operatorname{mad} .2020 .111316$

Dagher ZR, Erduran S (2014) Laws and explanations in biology and chemistry: philosophical perspectives and educational implications. In: Matthews MR (ed) International handbook of research in history, philosophy and science teaching. Springer Netherlands, Dordrecht, pp 1203-33

de Grey AD (2013) The desperate need for a biomedically useful definition of "aging." Rejuvenat Res 16:89-90. https://doi. org/10.1089/rej.2013.1428

Diamond JM (1982) Big-bang reproduction and ageing in male marsupial mice. Nature 298:115-116

Dilman VM, Revskoy SY, Golubev AG (1986) Neuroendocrine-ontogenetic mechanism of aging: toward an integrated theory of aging. Int Rev Neurobiol 28:89-156

Droge W (2003) Oxidative stress and aging. Adv Exp Med Biol 543:191-200

Dyre JC (2018) Isomorph theory of physical aging. J Chem Phys 148:154502. https://doi.org/10.1063/1.5022999

Faragher RGA (2015) Should we treat aging as a disease? The consequences and dangers of miscategorisation. Front Genet 6:171. https://doi.org/10.3389/fgene.2015.00171

Flatt T (2012) A new definition of aging? Front Genet 3:148

Franceschi C, Garagnani P, Morsiani C, Conte M, Santoro A, Grignolio A, Monti D, Capri M, Salvioli S (2018) The continuum of aging and age-related diseases: common mechanisms but different rates. Front Med. https://doi.org/ 10.3389/fmed.2018.00061

Gladyshev VN (2013) The origin of aging: imperfectness-driven non-random damage defines the aging process and control of lifespan. Trends Genet 29(9):506-512

Goldstein DS, Kopin IJ, Sharabi Y (2014) Catecholamine autotoxicity. Implications for pharmacology and therapeutics of Parkinson disease and related disorders. Pharmacol Therap 144:268-282. https://doi.org/10.1016/j. pharmthera.2014.06.006

Golubev AG (1996) The other side of metabolism: a review. Biochemistry-Moscow 61:1443-1460

Golubev A (2009) How could the Gompertz-Makeham law evolve. J Theor Biol 258:1-17. https://doi.org/10.1016/j. jtbi.2009.01.009

Golubev A (2012) Genes at work in random bouts. BioEssays 34:311-319

Golubev AG (2018) Is aging a disease? A biogerontologist's point of view: senescence $\neq$ disease. Adv Gerontol 8:127-129. https://doi.org/10.1134/s2079057018020066

Golubev AG (2018b) Commentary: is life extension today a Faustian bargain? Front Med. https://doi.org/10.3389/ fmed.2018.00073 
Golubev A (2019a) A 2D analysis of correlations between the parameters of the Gompertz-Makeham model (or law?) of relationships between aging, mortality, and longevity. Biogerontology 20:799-821. https://doi.org/10.1007/ s10522-019-09828-z

Golubev AG (2019b) Why and how do we age? A single answer to two questions. Adv Gerontol 9:1-14

Golubev AG (2020) COVID-19: a challenge to physiology of aging. Front Physiol. https://doi.org/10.3389/fphys.2020. 584248

Golubev A, Hanson AD, Gladyshev VN (2017a) Non-enzymatic molecular damage as a prototypic driver of aging. J Biol Chem 292:6029-6038. https://doi.org/10.1074/jbc. R116.751164

Golubev A, Hanson AD, Gladyshev VN (2017b) A tale of two concepts: harmonizing the free radical and antagonistic pleiotropy theories of aging Antiox Redox. Signal 29:1003-1017. https://doi.org/10.1089/ars.2017.7105

Golubev A, Panchenko A, Anisimov V (2018) Applying parametric models to survival data: tradeoffs between statistical significance, biological plausibility, and common sense. Biogerontology 19:341-365. https://doi.org/10.1007/ s10522-018-9759-3

Gorisse L, Pietrement C, Vuiblet V, Schmelzer CEH, Köhler M, Duca L, Debelle L, Fornès P, Jaisson S, Gillery P (2016) Protein carbamylation is a hallmark of aging. Proc Natl Acad Sci USA 113:1191-1196. https://doi.org/10.1073/ pnas. 1517096113

Gupta A (2019) Definitions. In: Zalta N (ed) The Stanford Encyclopedia of Phylosophy. Metaphysics Research Lab, Stanford University. https://plato.stanford.edu/archives/ win2019/entries/definitions

Hamilton WD (1966) The moulding of senescence by natural selection. J Theor Biol 12:12-45. https://doi.org/10.1016/ 0022-5193(66)90184-6

Harman D (1992) Free radical theory of aging. Mutat Res 275:257-266

Hassani OK, Rymar VV, Nguyen KQ, Huo L, Cloutier J-F, Miller FD, Sadikot AF (2020) The noradrenergic system is necessary for survival of vulnerable midbrain dopaminergic neurons: implications for development and Parkinson's disease. Neurobiol Aging 85:22-37. https://doi.org/10. 1016/j.neurobiolaging.2019.09.014

Hawley JA, Joyner MJ, Green DJ (2021) Mimicking exercise: what matters most and where to next? J Physiol 599:791-802. https://doi.org/10.1113/JP278761

Hayflick L (2007) Entropy explains aging, genetic determinism explains longevity, and undefined terminology explains misunderstanding both. PLoS Genet 3(12):e220

Hayflick L (2021) The greatest risk factor for the leading cause of death is ignored. Biogerontology 22:133-141

Hong SY, Ng LT, Ng LF, Inoue T, Tolwinski NS, Hagen T, Gruber J (2016) The role of mitochondrial non-enzymatic protein acylation in ageing. PLoS ONE 11:e0168752. https://doi.org/10.1371/journal.pone.0168752

Jacobs HIL, Riphagen JM, Ramakers IHGB, Verhey FRJ (2019) Alzheimer's disease pathology: pathways between central norepinephrine activity, memory, and neuropsychiatric symptoms. Mol Psychiatry. https://doi.org/10.1038/ s41380-019-0437-X
Kaeberlein M (2017) Translational geroscience: a new paradigm for 21 st century medicine. Transl Med Aging 1:1-4. https://doi.org/10.1016/j.tma.2017.09.004

Kennedy BK, Pennypacker JK (2014) Drugs that modulate aging: the promising yet difficult path ahead. Transl Res 163:456-465. https://doi.org/10.1016/j.trsl.2013.11.007

Kennedy BK, Berger SL, Brunet A, Campisi J, Cuervo AM, Epel ES, Franceschi C, Lithgow GJ, Morimoto RI, Pessin JE, Rando TA, Richardson A, Schadt EE, Wyss-Coray T, Sierra F (2014) Geroscience: linking aging to chronic disease. Cell 159:709-13. https://doi.org/10.1016/j.cell. 2014.10.039

Kold-Christensen R, Johannsen M (2020) Methylglyoxal metabolism and aging-related disease: moving from correlation toward causation. Trends Endocrinol Metab 31:81-92. https://doi.org/10.1016/j.tem.2019.10.003

Kövecses Z (2020) A brief outline of "standard" conceptual metaphor theory and some outstanding issues. Extended conceptual metaphor theory. Cambridge University Press, Cambridge, pp 1-21

Kowald A, Kirkwood TB (2016) Can aging be programmed? A critical literature review. Aging Cell 15:986-998. https:// doi.org/10.1111/acel.12510

Krashia P, Nobili A, D'Amelio M (2019) Unifying hypothesis of dopamine neuron loss in neurodegenerative diseases: focusing on Alzheimer's disease. Front Mol Neurosci. https://doi.org/10.3389/fnmol.2019.00123

Laplane L, Mantovani P, Adolphs R, Chang H, Mantovani A, McFall-Nga M et al (2019) Opinion: why science needs philosophy. Proc Natl Acad Sci USA 116(10):3948-52

Larsen SA, Kassem M, Rattan SIS (2012) Glucose metabolite glyoxal induces senescence in telomerase-immortalized human mesenchymal stem cells. Chem Cent J 6:18. https:// doi.org/10.1186/1752-153X-6-18

Lemoine M (2020) Defining aging. Biol Philos 35:46. https:// doi.org/10.1007/s10539-020-09765-Z

Lerma-Ortiz C, Jeffryes JG, Cooper AJL, Niehaus TD, Thamm AMK, Frelin O, Aunins T, Fiehn O, de Crécy-Lagard V, Henry CS, Hanson AD (2016) 'Nothing of chemistry disappears in biology': the Top 30 damage-prone endogenous metabolites. Biochem Soc Trans 44:961-971. https://doi. org/10.1042/bst20160073

Lewis D (1970) How to define theoretical terms. J Philos 67:427-446

López-Otín C, Blasco MA, Partridge L, Serrano M, Kroemer G (2013) The hallmarks of aging. Cell 153:1194-1217. https://doi.org/10.1016/j.cell.2013.05.039

Lyons TJ, Jenkins AJ (1997) Glycation, oxidation, and lipoxidation in the development of the complications of diabetes: a carbonyl stress hypothesis. Diabetes Rev (Alexandria, Va.) 5:365

Marck A, Antero J, Berthelot G, Saulière G, Jancovici J-M, Masson-Delmotte V, Boeuf G, Spedding M, Le Bourg É, Toussaint J-F (2017) Are we reaching the limits of homo sapiens? Front Physiol. https://doi.org/10.3389/fphys. 2017.00812

Martel J, Chang S-H, Wu C-Y, Peng H-H, Hwang T-L, Ko Y-F, Young JD, Ojcius DM (2021) Recent advances in the field of caloric restriction mimetics and anti-aging molecules. Ageing Res Rev 66:101240. https://doi.org/10.1016/j.arr. 2020.101240 
Martin GM (1996) Somatic mutagenesis and antimutagenesis in aging research. Mutat Res 350(1):35-41

McCann SM, Mastronardi C, de Laurentiis A, Rettori V (2005) The nitric oxide theory of aging revisited. Ann NY Acad Sci 1057:64-84. https://doi.org/10.1196/annals.1356.064

Moger-Reischer RZ, Lennon JT (2019) Microbial ageing and longevity. Nat Rev Microbiol 17:679-690. https://doi.org/ 10.1038/s41579-019-0253-y

Muchowska KB, Varma SJ, Moran J (2019) Synthesis and breakdown of universal metabolic precursors promoted by iron. Nature 569:104-107. https://doi.org/10.1038/s41586019-1151-1

Muise ES, Guan H-P, Liu J, Nawrocki AR, Yang X, Wang C, Rodríguez CG, Zhou D, Gorski JN, Kurtz MM, Feng D, Leavitt KJ, Wei L, Wilkening RR, Apgar JM, Xu S, Lu K, Feng W, Li Y, He H, Previs SF, Shen X, van Heek M, Souza SC, Rosenbach MJ, Biftu T, Erion MD, Kelley DE, Kemp DM, Myers RW, Sebhat IK (2019) Pharmacological AMPK activation induces transcriptional responses congruent to exercise in skeletal and cardiac muscle, adipose tissues and liver. PLoS ONE 14:e0211568. https://doi.org/ 10.1371/journal.pone.0211568

Peana AT, Bassareo V, Acquas E (2019) Not just from ethanol. Tetrahydroisoquinolinic (TIQ) derivatives: from neurotoxicity to neuroprotection. Neurotox Res 36:653-668. https://doi.org/10.1007/s12640-019-00051-9

Peto R, Parish SE, Gray RG (1985) There is no such thing as ageing, and cancer is not related to it. IARC Sci Publ 58:43-53

Pimm D (2014) Authority, explanation, contention and register: language data and the surface search for essence. ZDM Math Educ 46:967-976. https://doi.org/10.1007/s11858014-0633-8

Ralser M (2018) An appeal to magic? The discovery of a nonenzymatic metabolism and its role in the origins of life. Biochem J 475:2577-2592. https://doi.org/10.1042/ bcj20160866

Rando TA, Wyss-Coray T (2021) Asynchronous, contagious and digital aging. Nat Aging 1:29-35. https://doi.org/10. 1038/s43587-020-00015-1

Rattan SIS (2012) Biogerontology: from here to where? The Lord Cohen Medal Lecture-2011. Biogerontology 13:83-91. https://doi.org/10.1007/s10522-011-9354-3

Rattan S (2016) If Aging is a disease, then it is your own fault. J Aging Sci 4:2. https://doi.org/10.4172/2329-8847. $1000 \mathrm{e} 120$

Rattan SIS (2020) Naive extrapolations, overhyped claims and empty promises in ageing research and interventions need avoidance. Biogerontology 21:415-421

Ravichandran M, Priebe S, Grigolon G, Rozanov L, Groth M, Laube B, Guthke R, Platzer M, Zarse K, Ristow M (2018) Impairing L-threonine catabolism promotes healthspan through methylglyoxal-mediated proteohormesis. Cell Metab 27:914-925.e915. https://doi.org/10.1016/j.cmet. 2018.02.004

Sadowska-Bartosz I, Bartosz G (2014) Effect of antioxidants supplementation on aging and longevity. BioMed Res Int 2014:17. https://doi.org/10.1155/2014/404680

Sadowska-Bartosz I, Bartosz G (2016) Effect of glycation inhibitors on aging and age-related diseases. Mech Ageing Dev 160:1-18. https://doi.org/10.1016/j.mad.2016.09.006
Samaras TT (1974) The law of entropy and the aging process. Hum Dev 17:314-320

Schenkelaars Q, Tomczyk S, Wenger Y, Ekundayo K, Girard V, Buzgariu W, Austad S, Galliot B (2018) Chapter 38-hydra, a model system for deciphering the mechanisms of aging and resistance to aging. In: Ram JL, Conn PM (eds) Conn's handbook of models for human aging, 2nd edn. Academic Press, Cambridge, pp 507-520

Semchyshyn HM (2014) Reactive carbonyl species in vivo: generation and dual biological effects. Sci World J 2014:417842. https://doi.org/10.1155/2014/417842

Sies H, Jones DP (2020) Reactive oxygen species (ROS) as pleiotropic physiological signalling agents. Nat Rev Molec Cell Biol. https://doi.org/10.1038/s41580-020-0230-3

Simic Z, Weiwad M, Schierhorn A, Steegborn C, Schutkowski $M$ (2015) The -amino group of protein lysine residues is highly susceptible to nonenzymatic acylation by several physiological Acyl-CoA thioesters. ChemBioChem 16:2337-2347. https://doi.org/10.1002/cbic.201500364

Strehler BL, Mildvan AS (1960) General theory of mortality and aging. Science 132:14-21

Stroustrup N (2018) Measuring and modeling interventions in aging. Curr Opin Cell Biol 55:129-138. https://doi.org/10. 1016/j.ceb.2018.07.004

Taghavi F, Habibi-Rezaei M, Amani M, Saboury AA, MoosaviMovahedi AA (2016) The status of glycation in protein aggregation. Int J Biol Macromol. https://doi.org/10.1016/ j.ijbiomac.2015.12.085

Tarkhov AE, Menshikov LI, Fedichev PO (2017) StrehlerMildvan correlation is a degenerate manifold of Gompertz fit. J Theor Biol 416:180-189. https://doi.org/10.1016/j. jtbi.2017.01.017

Tomás-Loba A, Flores I, Fernández-Marcos PJ, Cayuela ML, Maraver A, Tejera A, Borrás C, Matheu A, Klatt P, Flores JM, Viña J, Serrano M, Blasco MA (2008) Telomerase reverse transcriptase delays aging in cancer-resistant mice. Cell 135:609-622. https://doi.org/10.1016/j.cell.2008.09. 034

Tran QP, Adam ZR, Fahrenbach AC (2020) Prebiotic reaction networks in water. Life 10:352

Wang G, Zhou Y, Wang Y, Li D, Liu J, Zhang F (2019) Ageassociated dopaminergic neuron loss and midbrain glia cell phenotypic polarization. Neuroscience 415:89-96. https:// doi.org/10.1016/j.neuroscience.2019.07.021

Weinshenker D (2018) Long road to ruin: noradrenergic dysfunction in neurodegenerative disease. Trends Neurosci 41:211-223. https://doi.org/10.1016/j.tins.2018.01.010

Wołos A, Roszak R, Żądło-Dobrowolska A, Beker W, MikulakKlucznik B, Spólnik G, Dygas M, Szymkuć S, Grzybowski BA (2020) Synthetic connectivity, emergence, and selfregeneration in the network of prebiotic chemistry. Science 369:eaaw1955. https://doi.org/10.1126/science.aaw1955

Wu Z, Fu Y, Yang Y, Huang C, Zheng C, Guo Z, Yang Z, Chen X, Zhu J, Wang J, Li X, Chen L, Zhou W, Chen Y, Wang J, Yang Y, Jiang M, Chen S, Lu A, Liu J, Li Y, Sun S, Wang Z, Xiao W, Wang Y (2018) Methylglyoxal modification of TrkB promotes synaptic plasticity and enhances resilience to chronic stress. bioRxiv. https://doi.org/10.1101/435867

Yang Y, Santos AL, Xu L, Lotton C, Taddei F, Lindner AB (2019) Temporal scaling of aging as an adaptive strategy of 
Escherichia coli. Sci Adv 5:eaaw2069. https://doi.org/10. 1126/sciadv.aaw2069

Zhavoronkov A, Bhullar B (2015) Classifying aging as a disease in the context of ICD-11. Front Genet. https://doi.org/10. 3389/fgene.2015.00326
Publisher's Note Springer Nature remains neutral with regard to jurisdictional claims in published maps and institutional affiliations. 